\title{
Individual Selection for Cooperative Group Formation
}

\author{
Simon T. Powers, Alexandra S. Penn, and Richard A. Watson \\ School of Electronics and Computer Science, University of Southampton, U.K.; \\ stp05r@ecs.soton.ac.uk
}

\begin{abstract}
It is well known that certain environmental conditions, such as a spatially structured population, can promote the evolution of cooperative traits. However, such conditions are usually assumed to be externally imposed. In this paper, we present a model that allows the conditions that promote or hinder cooperation to arise adaptively via individual selection. Consequently, instead of selection simply favouring cooperation under imposed environmental conditions, in our model selection also operates on the conditions themselves via a niche construction process. Results are presented that show that the conditions that favour cooperation can evolve, even though those that favour selfish behaviour are also available and are initially selected for.
\end{abstract}

\section{Introduction}

In this paper, we consider the evolution of cooperative behaviour that benefits all individuals within a group. An example of such behaviour is bacteria growing at a reduced rate in order to consume a limiting resource more efficiently [14]. The difficulty in explaining the evolution of such behaviour arises from the fact that it can be exploited by selfish cheaters who do not pay the costs of performing the cooperative act themselves but nevertheless still reap the group benefit. In the bacterial growth example, cheaters would be individuals who unilaterally grow at a faster rate but in so doing waste resource. If resource is shared freely between all members of the group, then cheaters will be favoured under individual selection. This follows since all members of the group feel the effects of their inefficient resource usage, while only they grow at a faster rate.

However, environmental conditions such as a spatially structured population can allow prolonged cooperation to be maintained under individual selection [5, 1-3]. As an extreme example, if an individual only shares resource with itself then selection will clearly favour individuals that consume the limiting resource with less wastage. A more common situation is that the existence of spatial structure provides a setting where an individual only shares resource with a small number of its neighbours. Such a setting allows the formation of clusters of neighbouring cooperators that share the majority of their resource only with each other. Consequently, the cooperators in the cluster do not feel the effects of the wasteful resource usage of cheaters that are located elsewhere in the population, and so will ultimately reach a larger biomass in the long term [1-3]. 
Unfortunately, such a cluster of cooperators could still be exploited by the appearance of a mutant cheater amongst their ranks [3]. Therefore, the existence of spatial structure alone is not sufficient to purge cheaters. Indeed, it has already been proposed in this resource sharing scenario that clusters of cooperators should periodically break up into single cells in order to prevent them from being outcompeted by mutant or immigrant cheaters [4]. A somewhat similar approach that can also purge cheaters is Wilson's well known trait-group aggregation and dispersal model [6-8]. This is a model based on the idea that groups containing a greater proportion of cooperators grow to a larger size than those containing more cheaters. If the progeny of the groups are then periodically mixed, and there is sufficient difference in group sizes after growth to counter the decline in frequency of cooperators within any mixed groups in which they are exploited, then this can lead to a global increase in the frequency of cooperators. This outcome can be explained in one of two ways. One view is that selection acting between groups (differential group productivity) favours cooperation, while selection within groups favours cheating; the end result is then determined by the balance of these two selective forces. The alternative, reductionist, view point is that the cooperative trait can have the greater individual fitness in the context of a group aggregation and dispersal population structure. Both of these viewpoints are compatible with each other, since neither denies that the allele that increases in frequency globally is the one with the highest fitness when averaged across the whole population [8].

One of the key determinants of whether the cooperative trait does in fact have a greater fitness is the variation in final group sizes before the groups are remixed, since this must be sufficient to outweigh selection operating within groups that favours cheaters. This in turn is affected by the variation in group composition when the groups are formed. For example, it has already been shown that assortative grouping, whereby a propensity exists for individuals to form groups with other playing the same strategy, facilitates cooperation in cases where random group formation is insufficient $[7,9]$. Another way of increasing initial variance in group composition that is applicable to random group formation is to reduce the initial group size, thus increasing the sampling error on the global population due to randomly formed groups.

The above discussion has shown how cooperation can be favoured by certain environmental conditions or modelling choices. However, in previous works, the parameter settings that give the cooperative strategy the advantage under individual selection, e.g. small group size in an aggregation and dispersal model, have been assumed. As a result, the explanatory power of the models has been limited to revealing the conditions under which cooperation is favoured. Consequently, they have left unanswered the following question. If individuals can modify the environmental conditions through individual adaptation such that either cooperative or selfish behaviour is favoured, which conditions and hence which behaviour will evolve? Thus, rather than imposing the environmental conditions that favour cooperation, in this paper we allow individual adaptation to determine whether or not these conditions arise. This modification of environ- 
mental conditions by the evolving individuals is an evolutionary dynamic known as niche construction [10], and is discussed further in Sect. 4.

In our model, we allow the initial group size parameter of the aggregation and dispersal process to be brought under individual control. Specifically, individuals posses a genotype that carries two parameters. The first of these is whether or not they grow at a reduced rate in order to use a limiting resource more efficiently, i.e. whether they are cooperative or selfish. The second parameter specifies the size of group that they will join, large or small. Our model also contains an intrinsic advantage to large groups, in the form of a greater per capita resource allocation.

It should be noted that individual choice over group size is realistic in many biological settings. For example, bacteria are known to be able to control microcolony size in biofilms via the amount of Extracellular Polymeric Substances that they secrete and the process of quorum sensing. This example illustrates the fact that high-level cognitive abilities are not required in order for group size to be determined adaptively by individuals.

Using our model, we obtain results in which the following pairwise relationships hold: cooperative + large outcompetes cooperative + small, and selfish + large outcompetes selfish + small. Therefore, evolution should be towards the large initial group size environmental state. It is then also the case that selfish + large outcompetes cooperative + large. This leads to the conclusion that the direction of evolution should be towards large groups of selfish individuals. However, when all 4 genotypes are present in the initial population in equal proportions, this is not what happens. Instead, evolution leads to the fixation of the cooperative + small genotype. Therefore, although pairwise comparisons suggest that selfish + large is favoured under individual selection, the dynamics are such that when all 4 strategies are present it is cooperative + small that wins. We therefore show in this paper that, given certain parameter settings and assumptions, individual adaptation of environmental conditions can lead to those that favour cooperation, even though it initially seems that the opposite environmental conditions and strategy should be promoted under individual selection.

\section{Model Details}

Our model consists of a population of individuals that reproduce in randomly formed groups for a number of generations $(t)$, before the progeny of each group are mixed together into a migrant pool and the process repeated. The genotype of each individual specifies two traits:

1. Cooperative or selfish resource usage (specified as a growth rate, $G_{i}$, and a resource consumption rate $C_{i}$ );

2. specification of the initial size of the group that that the individual will join (small or large). 
We therefore consider 4 possible genotypes / strategies: cooperative + small, cooperative + large, selfish + small and selfish + large.

In our model, reproduction occurs entirely within groups, as follows. Let $n_{i}$ represent the number of copies of genotype $i$ in a single group. In order to reproduce, these clones require a share of the group's resource influx, $R$. The amount of resource that a group receives at each time-step depends upon its size, with a larger per capita amount of resource allocated to larger groups. Specifically, a group that is twice as large receives an extra $5 \%$ per capita resource influx. The size of this share that each genotype receives depends upon its growth and consumption rates relative to those of the other genotype(s), where the consumption rate should be understood as the amount of resource required for a genotype to make one copy of itself. The magnitude of the share of the total group resource that the genotype receives, $r_{i}$, is then as defined in (1).

$$
r_{i}=\frac{n_{i} G_{i} C_{i}}{\sum_{j}\left(n_{j} G_{j} C_{j}\right)} R
$$

Therefore, the genotype with the highest growth and consumption rates will receive the largest per capita share of the total resource. This means that the selfish genotype always receives more per capita resource than the cooperative type, and so will ultimately drive a cooperative genotype in the same group extinct.

Given the share received by a genotype, the number of individuals in the group with that genotype then changes according to (2). The form of this equation is motivated as a simplification of existing models of bacterial colony growth $[1-3]$.

$$
n_{i}(t+1)=n_{i}(t)+\frac{r_{i}}{C_{i}}-K n_{i}(t)
$$

As the replicator equation (2) shows, the number of clones of a genotype produced during reproduction depends on both growth (favouring the selfish type in the short-term) and consumption rates (favouring the cooperative type in the long-term under limited resource). The final term in (2) represents mortality, where $K$ is a death rate that is constant to all genotypes.

The remainder of our model is based around Wilson's trait-group aggregation and dispersal process. In order to address our research question, the model differs from others that use this process in that in our model individuals carry a gene that determines the initial size of group that they join. Consequently, this parameter can be set adaptively during evolution. By contrast, in other models the value of this, and all other parameters of the aggregation and dispersal process, are imposed. Thus, in those models the conditions that determine whether or not cooperative behaviour is selected for are imposed, whereas in our model they are determined by the individuals themselves.

The other ways in which our model differs from the original trait-group aggregation and dispersal process [6] are that reproduction occurs within groups and that we impose both global, and group, carrying capacities, where the group 
carrying capacity follows from a finite resource influx. The overall algorithmic operation of our model is presented below:

1. Initialisation: Initialise the migrant pool with $N$ individuals.

2. Group formation (aggregation): Assign individuals in the migrant pool to groups, as described in the main text below.

3. Reproduction: Perform reproduction within groups for $t$ time-steps, as described in the text above.

4. Migrant pool formation (dispersal): Return the progeny of each group to the migrant pool.

5. Maintaining the global carrying capacity: Rescale the migrant pool back to size $N$, retaining the proportion of individuals with each genotype.

6. Iteration: Repeat from step 2 onwards for a number of generations, $T$.

Assignment of individuals to groups occurs by the following process. Groups of the "small" size are created by choosing (without replacement) individuals at random who specify the small size parameter on their genotype. Likewise, groups of the "large" size are created from randomly drawn individuals who specify the large parameter. Therefore, although all individuals in a group will specify the same size trait, the composition of the group will be random with respect to the cooperative / selfish resource usage trait. Finally, if there are an insufficient number of individuals remaining in the migrant pool to form the appropriate group size, then those remaining unassigned to groups are discarded. Given a large population size, the effect of this is negligible.

\section{Results}

In this section, we first investigate the results of pairwise competition between strategies, before proceeding to investigate the dynamics that occur when all 4 strategies are available. Throughout this section, the parameter settings stated in Table 1 are used.

Table 1. Parameter settings used throughout.

\begin{tabular}{|l|l|}
\hline Parameter & Value \\
\hline Growth rate (cooperative), $G_{c}$ & 0.018 \\
\hline Growth rate (selfish), $G_{s}$ & 0.02 \\
\hline Consumption rate (cooperative), $C_{c}$ & 0.1 \\
\hline Consumption rate (selfish), $C_{s}$ & 0.2 \\
\hline Population size, $N$ & 4000 \\
\hline Number of generations, $T$ & 1000 \\
\hline
\end{tabular}




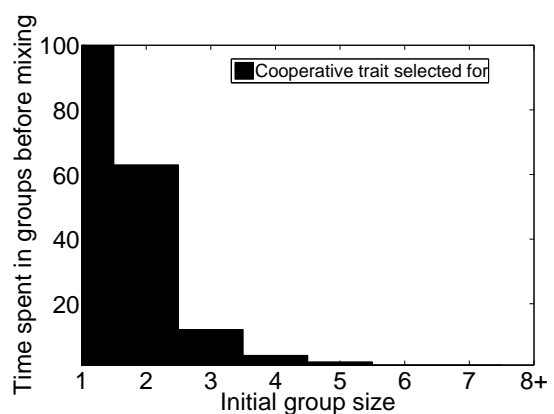

Fig. 1. Equilibrium state as a function of group size and the time spent within groups. The black area indicates the region of this parameter space where cooperation is favoured; the white area the region where selfish cheating is favoured.

\subsection{Preliminary Experimentation / Pairwise Competition}

When deciding on values for "small" and "large" group sizes, it was essential that the imposition of the small size upon all members of the population lead to selection favouring the cooperative trait, and the imposition of the large size lead to selection favouring the selfish trait. This would then give individuals the possibility of adaptively determining whether the environmental conditions, in the form of group size, lead to cooperation being selected for.

To determine suitable values for large and small group sizes, the 2D parameter space consisting of group size and the time spent in groups prior to mixing (returning to the migrant pool) was sampled; all other parameters of the model were held constant. From this sampling, it was possible to plot the parameter space with respect to whether cooperation was selected for, as shown in Fig. 1. Note that cooperation is reported as being selected for if and only if the cooperative trait reaches fixation in the global population at equilibrium.

As expected, this sample of the space shows that cooperation is favoured by both small groups and a short time spent within those groups prior to mixing. Small groups favour cooperation by increasing the variance in group composition, thereby strengthening selection acting between groups, i.e. selection that is due to the difference in group sizes after reproduction [8]. A short time spent in groups prior to mixing favours cooperation since the longer the time spent in groups, the greater the decline in frequency of cooperators within all groups that also contain cheaters [8].

Given the results in Fig. 1, it was decided to set the small group size at 4 and the large at 40 , with the time spent within groups fixed at 4 . This then creates the situation in which cooperative + small outcompetes selfish + small, and selfish + large outcompetes cooperative + large. Therefore, our definition of small size favours cooperative efficient resource usage, while our definition of large favours selfish inefficient usage.

However, further preliminary results showed that in pairwise competition, cooperative + small is outcompeted by cooperative + large. This is due to the 

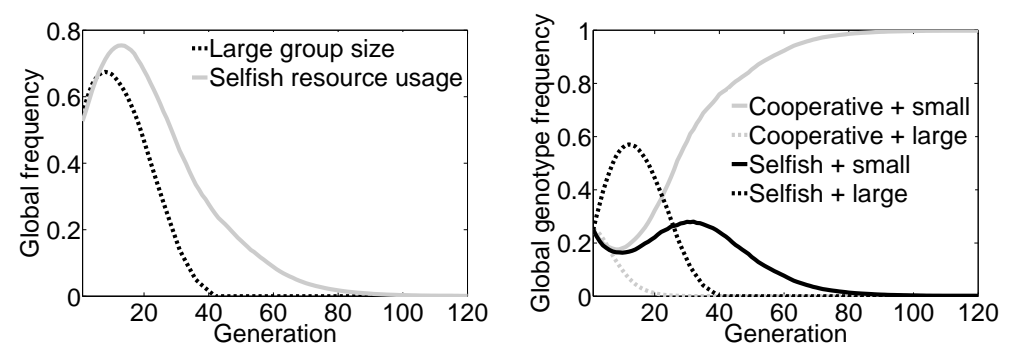

Fig. 2. Left-hand plot: average environment and strategy through time. Right-hand plot: change in genotype frequencies over time.

greater per capita resource influx provided to large groups, which allows such groups to grow to a larger size, assuming all other conditions are the same. Likewise, selfish + large outcompetes selfish + small. Given that large genotypes outcompete their small counterparts, and that in an environment with large groups selfish resource usage is favoured, it should be expected that the population would evolve towards a state with the selfish + large genotype at fixation. However, the results in the next section show that this is not the case if the population is initialised with all 4 genotypes in equal proportion.

\subsection{Allowing Both Strategy and Environment to Evolve}

The left-hand plot in Fig. 2 shows how the average environmental conditions, in terms of group size, and the average resource usage strategy, change over time. The key point is that at the start neither small groups nor cooperators are favoured; this is shown by an increase in the frequency of both large groups and selfish cheaters. Therefore, since the initial conditions favour both large groups and cheaters, it might be expected that the equilibrium reached would consist entirely of large groups of cheaters. However, this is not what occurs. Instead, after around 20 generations, both small groups and cooperators become favoured by selection. Therefore, the selective pressures change in a way that could not be predicted from pairwise comparisons alone.

The right-hand plot in Fig. 2 can help to explain why this occurs, by showing how all possible combinations of environment and strategy change over time. The figure shows that initially, the environment of large groups is favoured; this is due to their per capita resource advantage. In such large groups, the selfish inefficient resource usage strategy is favoured, as previously discussed.

However, the selfish + large genotype does not reach fixation. This is because selfish cheaters benefit from consuming resource in mixed groups with cooperators, since they can consume the left-over resource that follows from the cooperators efficiency. As the selfish + large type increases in frequency, it reduces the frequency of cooperative + large and hence the number of cooperators that it can exploit. However, there are still cooperative + small individuals present in the population. This then creates a selective advantage of being in small groups 
which still contain cooperators that can be exploited, which explains the increase in frequency of both small types after around 10 generations. Finally, once small becomes the dominant size allele, the cooperative strategy wins, due to the fact that cooperators drive selfish cheaters extinct where the initial group size is 4 and $t=4$ (as in Sect. 3.1).

\section{Discussion}

The decision to live in groups of a certain size can be considered as an example of niche construction. It is commonly held that synergistic fitness effects in a cooperative, group-living scenario would have been an important driver in the formation of higher levels of biological organisation [11]. The potential of higher fitness within groups would have been an incentive for individuals to actively choose to participate in these groups bringing an aspect of their selective environment under control and altering their fitness landscape accordingly. Choosing to live within a group via some individual-level adaptation or adaptations that facilitate group formation would thus constitute the creation of a niche. This of course does not negate the problem of invasion or disruption by selfish individuals. As discussed earlier, in the absence of assortative groupings, cooperation within a group context is only stable when groups are small enough that between group variation can be maintained by sampling error, hence allowing between group selection to be an effective force. We might expect then that cooperative groups would be stable if the individuals within them were able to adaptively choose a group size small enough to purge selfish strategies from the population but large enough to enjoy the fitness benefits of group living.

The significance and originality of our model lies in the fact that the conditions that favour cooperation are not assumed. Instead, individuals in the population carry a specification of the conditions, in this case initial group size, on their genotype. Whether or not conditions that favour cooperation occur is then determined adaptively by individual selection on this specification. Figure 3 illustrates the two-way interaction of strategy and environment in this scenario. On the one hand, whether cooperative or selfish behaviour is preferred by individual adaptation is influenced by the environment - this is normal, environmentally sensitive, adaptation. On the other, whether individual adaptation will support the construction of environments with large groups or small groups is influenced by the strategy being played - this is niche construction. A system with these two influences must reach an attractor where the characteristics of the environment favour strategies that in turn support those particular environmental characteristics. In this case we need not presuppose, for example, that the prevailing environment favours cooperation in order for cooperation to arise; and similarly, we need not presuppose that the prevailing strategy is cooperative in order for cooperation-favouring groups to arise.

Previous models have shown that cooperative traits are selected for when environmental conditions favour cooperation. In our model, conditions that favour either selfish or cooperative traits are available. We have then been able to show 


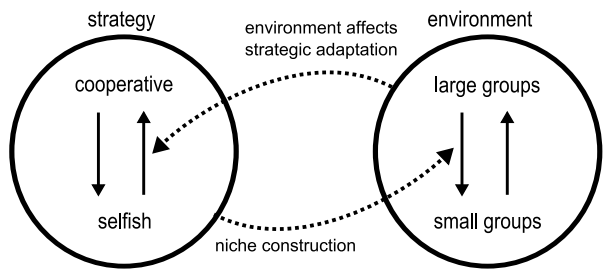

Fig. 3. Feedback between strategy and environment and their adaptation.

that, under given parameter settings and model assumptions, the conditions that promote cooperation (a small group size) are indeed selected for. This also occurs despite a bias towards the conditions (large groups) that favour selfish individuals. The result that the cooperative + small genotype reaches fixation, even though cooperative + large beats it in pairwise competition, is particularly interesting since a small group size can therefore be viewed as an adaptation that purges cheaters. The adaptive dynamics of the model can be seen more clearly in Fig. 2, which shows the changing global proportions of individuals choosing a large group environment and those choosing a selfish strategy over the course of the run. It is clear that both large group size and selfish resource use are initially favoured, however the frequency of the large group adaptation quickly peaks and starts to decline. In our scenario this occurs because a) in the presence of many large-group preferring cheaters, cooperators change their preference to favour small groups not large and the large-group cheaters become less and less fit; and, b) in the presence of many small cooperators, cheaters (also) change their preference to favour small groups not large. Once small groups are established, cooperation is the more fit strategy. This minimalist model illustrates the tightly coupled interaction of the evolution of strategy with the evolution of an individual's environment-affecting characters.

\section{Conclusion}

A plethora of models have shown that certain environmental conditions, such as a spatially structured population, can promote cooperative behaviour. This occurs because such conditions can give a cooperative trait a higher fitness than the selfish trait, when averaged over the whole population. Our contention however is that individuals are not simply the passive recipients of environmental conditions. Niche construction dynamics in which organisms adaptively alter their environments and thus their fitness landscapes have been observed in many systems and are postulated to be an important evolutionary dynamic [10]. It seems reasonable that individuals could adaptively choose to form or dissolve groups thus choosing the conditions that would facilitate either cooperative or selfish behaviour to be the preferred strategy. However, previous models have not considered the effect of allowing individual adaptation to determine whether or not these favourable conditions arise. 
In this paper, we have considered cooperation in the consumption of a limiting resource, where our cooperative strategy consumes resource more efficiently but suffers a cost of a reduced growth rate. This cost means that cooperators can be exploited by selfish individuals who consume resource less efficiently but that grow at a faster rate. However, it is already well known that Wilson's trait-group aggregation and dispersal model can potentially purge cheaters in situations such as this. One important parameter of the aggregation and dispersal process that determines whether or not cooperation wins out is the initial group size. Rather than impose this externally, as in previous models, we allow it to be set adaptively via individual selection in a process analogous to niche construction. In this minimal model we have demonstrated that when both cooperative and selfish strategies and the environmental conditions, the group size, which favours each, are both adaptable, then both cooperative behaviour and the small group size environment which actively supports it can evolve via individual selection even in conditions which initially favour large groups and selfish cheaters.

In future work, we may consider the introduction of new strategies via mutation. We also intend to examine in more detail the parameter values that promote cooperation and small groups, with regard to determining how large an attractor this equilibrium is. Finally, the process of niche construction should also be applicable to other parameters of the aggregation and dispersal process, for example, to the time spent reproducing in groups before mixing in the migrant pool. It would therefore be useful to investigate the individual adaptation of this and other parameters.

\section{References}

1. Pfeiffer, T., Schuster, S., Bonhoeffer, S.: Cooperation and competition in the evolution of ATP-producing pathways. Science 292(5516) (2001) 504-507

2. Pfeiffer, T., Bonhoeffer, S.: An evolutionary scenario for the transition to undifferentiated multicellularity. PNAS 100(3) (2003) 1095-1098

3. Kreft, J.U.: Biofilms promote altruism. Microbiology 150 (2004) 2751-2760

4. Kreft, J.U., Bonhoeffer, S.: The evolution of groups of cooperating bacteria and the growth rate versus yield trade-off. Microbiology 151 (2005) 637-641

5. Nowak, M.A., May, R.M.: The spatial dilemmas of evolution. International Journal of Bifurcation and Chaos 3(1) (1993) 35-78

6. Wilson, D.S.: A theory of group selection. PNAS 72(1) (1975) 143-146

7. Wilson, D.S.: The Natural Selection of Populations and Communities. Benjamin/Cummings (1980)

8. Sober, E., Wilson, D.S.: Unto Others: The Evolution and Psychology of Unselfish Behavior. Harvard University Press, Cambridge, MA (1998)

9. Wilson, D.S., Dugatkin, L.A.: Group selection and assortative interactions. The American Naturalist 149(2) (1997) 336-351

10. Odling-Smee, F.J., Laland, K.N., Feldman, M.W.: Niche construction: the neglected process in evolution. Monographs in population biology; no. 37. Princeton University Press (2003)

11. Keller, L., ed.: Levels of Selection in Evolution. Monographs in behavior and ecology. Princeton University Press (1999) 